The second point is the matter of visceral fat cells. We are happy that we were reminded of the excellent, careful work by Salans et al. ${ }^{1}$ These authors have as a matter of fact already studied this question very carefully. They studied the fat cell sizes at three subcutaneous and three visceral depots in fairly extensive material from obese and non-obese subjects. They then analysed their data from a number of different aspects, including calculations of fat cell number with and without visceral fat cells taken into account, and with different assumed percentages of visceral fat. Their conclusion was that ". . . mean adipose cell number for the obese group is always significantly greater than that for the non-obese, irrespective of the cell size used and of the assumed distribution of total body fat."

Not only the conclusions but also the results obtained are to a rather large extent different from those of Jung et al, and it is a pity that the reason for his has not been analysed in their report. The study by Salans et al shows conclusively that hypercellularity does exist. If we use the data of Jung et al, it does not exist if there are large difference in adipocyte distribution between subcutaneous and visceral fat in obese and non-obese subjects and this is a very unlikely situation.

Both these arguments demonstrate that the difference in opinion between our groups is actually quantitative. We believe that both groups agree on the basic point-that is, that adipose hypercellularity does exist, only its significance being evaluated differently. We do, of course, no use mean plus one $\mathrm{SD}$ of controls as cutting poin for definition of hyperplastic obesity other than for analytical purposes. ${ }^{2}$ In this report the low limit had to be set (arbitrarily, as stated in our paper) in order to get a large enough group of hypercellular patients. This again emphasises the quantitative aspects here: these are rare patients. We do, however, apparently see more such patients than Dr Jung and his colleagues, probably because we have a referring "filter," the very frequen everyday problem of slight obesity usually being taken care of outside our hospital. These severely obese subjects are, however, a considerable clinical problem although they are relatively few.

To sum up and close the discussion from our side, Dr Jung and his colleagues have brought up some important technical problems remaining in the field of adipocyte counting. We believe that we both think that adipose hypercellularity exists, although the division line is rather elusive and arbitrary just like the definition of obesity itself. We see more of the seriousness of the hyper cellularity problem than the English group does because we apparently get different patient material.

PER BJÖRNTORP LARS SJÖSTRÖM

Faculty of Medicine,
University of Göteborg,

Sweden

'Salans, L, Cushman, S W, and Weismann, R E, Fournal of Clinical Investigation, 1973, 52, 929. Nutrition, $1975, \mathbf{2 8}, 445$.
N

\section{Long-term parenteral nutrition}

SIR,-As directors of the Home Parenteral Nutrition Programme at the University of Washington, Seattle, we would like to make several comments regarding the article on home parenteral nutrition by Drs Karin Ladefoged and Stig Jarnum (22 July 1978, p 262). These comments are based on our own extensive experience over the last nine years. ${ }^{1}$

The authors do not make any differentiation between the complication rate for the standard subclavian vein catheter and the Broviac catheter. We have exclusively used the
Broviac catheter and have a much lower complication rate in our patients than the authors have reported. Specifically, in 137 catheters used in 94 patients with a tota infusion time of 1644 patient months, we have noticed only 23 episodes of associated septicaemia and five episodes of venous thrombosis. ${ }^{2}$ It is noteworthy that none of ou patients are on long-term anticoagulation.

The authors commented that unlike othe programmes they have found it difficult to maintain a standard parenteral nutrition infusion programme. This may be due to the unusually high percentage of patients that they have with jejunostomies. However, in our patients with short-bowel syndrome and either jejunostomies or ileostomies we keep the patients on a standard total parentera nutrition programme and supplement them with normal saline as needed to replace excessive gastrointestinal loss. We have been able to instruct our home patients to use two-channel infusion system-one channel fo nutrients is connected by a " $Y$ " to the other channel, which handles the normal saline.

Although some of our patients have been on home parenteral nutrition for over five years, we have seen vertebral compression fractures only in patients who have been on steroids. We have never seen significan osteomalacia with spontaneous bone fractures. However, we give patients 400 units of vitamin $\mathrm{D}$ daily, while the authors in this study gave them only 1200 units weekly. Perhaps their patients are therefore deficient in vitamin $D$.

DONALD G MiLleR MARIANNE IVEY BELDING H SCRIBNER

Division of Kidney Diseases,

University of Washington

School of Medicine,

Seattle, Washington 98195

Rault, R M J, and Scribner, B H, Progress in Gastroenterology, 1977, 3, 545 .

Miller, D G, et al, Surgery, Gynecology and Obstetrics, in press.

\section{Nutrition and cancer}

SIR,-Your leading article on malnutrition and cancer (7 April, p 912) reminds us that the cachexia of cancer is wrongly compared with the state of starvation. Starvation is a hypometabolic state in which there is a reduction in the turnover of carbohydrate, fat, and protein stores. ${ }^{1}$ Ketone bodies appear to play a major regulatory role. ${ }^{2}$ On the other hand, cancer cachexia is a hypermetabolic state $^{3}$ with increases in glycolysis, gluconeogenesis, lipolysis, and protein catabolism. ${ }^{45}$ Moreover, ketosis is an uncommon phenomenon in cancer patients (personal observations).

Using intravenous lipid solutions, we induced ketosis for eight days in a patient who had advanced hepatic cancer and peritoneal metastases. During that time she received only $56 \%$ of her usual calorie intake. However, she did not experience any change in her body weight, abdominal girth, or general well-being. Furthermore, she felt sated for the first time in months. Her serum albumin concentration gradually increased and her urinary and serum urea concentrations decreased, suggesting a reduction in endogenous protein catabolism. Neither hypoglycaemia nor lactic acidosis was observed.

Ketone bodies have been shown to reduce the growth, in culture, of transformed lymphoblasts from Burkitt's lymphoma and this effect is reversible, non-toxic, and proportional to the concentration of the ketone body up to $20 \mathrm{mM} .^{6}$ Normal cells are also known to have reduced cell growth rates in starvation. ${ }^{78}$ In leukaemia patients it has been shown that elevations in the proportion of immature cells in the bloodstream can be correlated with elevations in the basal metabolic rate. ${ }^{9}$

Surely these experimental findings raise the question: are forced feeding regimens for the treatment of advanced cancer metabolically sound? We have commenced a clinical tria to evaluate the role of hypometabolic (that is, ketotic) states in cancer therapy.

R A J CONYERS

A G NEED

A M ROFE

N POTEZNY

Institute of Medical and

Veterinary Science and

Adelaide, South Australia 5000

Cahill, G F, Clinical Endocrinology and Metabolism 1976, 5, 397.

Newsholme, E A, Clinical Endocrinology and Metabolism, 1976, 5, 543.

Cancer Res, A, and Silverstone, $\mathrm{H}$, Advances in Cancer Research, 1953, 1, 452.

Sciences, 1974, 230, 86 .
Scinal 1974, 230, 103 Magee, B, et al, in Proceedings of Australian Symposium on Nutrition and Cancer, 1978, $\mathrm{p} 25$

Aldewachi, et al, fournal of Anatomy, 1975, 119, 105. tragand, J J, and Hagemann, R F, American fournal of Clinical Nutrition, 1977, 30, 918

1921, 185, 785,

\section{Cancer after cardiac transplantation}

SIR,-Your leading article "Cancer after cardiac transplantation" (24 February, p 509) remarks: "The striking increase in the incidence of lymphoid neoplasms in patients who have transplants is intriguing but unexplained." It may therefore, be pertinent to draw attention to some animal models in which high incidences of malignant lymphoma were the long-term clinical result of a variety of experimental situations.

Armstrong et al trace the development of lymphomas associated with chronic allogeneic disease in mice as a "continuous transition from an immunological disorder, allogeneic disease, to a malignant growth . . . initiated by the transplantation of foreign lymphoid cells." Chronic antigenic stimulation of host tissues by allogeneic histocompatibility antigens resulted in neoplasia in this instance.

Another murine system excludes major histoincompatibilities by using syngeneic recipients. ${ }^{2}$ In this system malignant lymphoma has also been the sequel to syngeneic transplantations without immunosuppression. A high incidence of lymphoma $(60-70 \%)$ was the neoplastic outcome in syngeneic recipients of mammary gland and lymph node tissue, ${ }^{2}$ irrespective of the different treatments of the grafts before transplantation. Von Boehmer et $a l^{3}{ }^{4}$ reported that a syngeneic mixed lymphocyte reaction in mice between thymus cells and peripheral lymphocytes indicates the presence of antigenic differences between syngeneic lymphocytes even without a difference at a major histocompatibility locus. These authors postulate the presence of an antigen on peripheral lymphocytes not coded for by the major histocompatibility complex. Chronic stimulation of host lymphoid tissue by this antigen may play a part in the development of lymphoma in syngeneic graft recipients.

Even in autologous hosts it was possible to induce malignant lymphoma ${ }^{5}$ by first exposing the 